\title{
Editorial
}

\section{Hypothyroidism, thyroxine treatment, and the heart}

Hypothyroidism is a common condition; surveys indicate that approximately $1 \%$ of the general population and $4 \%$ of people 60 years and older are prescribed thyroxine long term. ${ }^{1}$ Hypothyroidism has cardiovascular consequences resulting from both direct influences of thyroid hormone deficiency on the heart, and adverse effects on circulating lipid concentrations. Furthermore, with the advent of improved tests of thyroid function, it has become clear that even when patients with hypothyroidism are treated with thyroxine long term, about half have serum thyrotrophin concentrations above or below the normal range, indicating a degree of under or over treatment with thyroxine. Although little importance has been attached to these minor biochemical abnormalities, recent evidence suggests that they may have considerable clinical significance.

Cardiovascular effects of untreated hypothyroidism Hypothyroidism is associated with sinus bradycardia, 24 hour ambulatory studies revealing decreases in resting, mean, and maximal heart rates. ${ }^{2}$ These reductions in heart rate and atrioventricular conduction blocks are the only dysrhythmias recognised to be associated with hypothyroidism, which may itself reduce the likelihood of ventricular dysrhythmias. ${ }^{3}$ An increase in diastolic blood pressure is also well described in hypothyroidism, and may be present in as many as $20 \%$ of subjects. ${ }^{4}$ This diastolic hypertension is in turn associated with an increase in peripheral vascular resistance. Myocardial contractility is impaired with a reduction in resting left ventricular ejection fraction; diastolic function may also be impaired, contributing to a reduction in cardiac output, ${ }^{5}$ and in a few cases to the development of heart failure. Pericardial effusions, demonstrated by echocardiography, are frequent in marked thyroid hormone deficiency, although tamponade is rare.

Untreated hypothyroidism is associated with hyperlipidaemia, specifically with increases in circulating concentrations of total and low density lipoprotein cholesterol, and decreases in high density lipoprotein cholesterol. ${ }^{6}$ It is often stated that ischaemic heart disease is more frequent in patients with hypothyroidism; this may be the case, but evidence in support of this view is scanty. A necropsy study demonstrated more coronary atherosclerosis in hypertensive hypothyroid patients than in hypertensive euthyroid subjects, but no difference was found between normotensive hypothyroid and euthyroid groups. ${ }^{7}$ A further necropsy study reported more "severe" coronary atherosclerosis in hypothyroid than euthyroid subjects, but neither study demonstrated a difference in prevalence of myocardial infarction. ${ }^{8}$ This suggests that hypothyroidism may protect against myocardial ischaemia, a view which in the past led to the use of radioiodine in the treatment of angina.

\section{Effects of thyroxine treatment}

Many of the cardiovascular abnormalities of hypothyroidism are corrected by thyroxine replacement therapy. Increases in heart rate occur within days of beginning thyroxine therapy. A fall in diastolic blood pressure is found in many, and abnormalities of ventricular systolic and diastolic function are also corrected by thyroxine replacement. ${ }^{5}$

Despite these clear benefits, it is well known that angina and myocardial infarction may be precipitated by the initiation of thyroxine replacement treatment (even in low dose) in those with underlying coronary artery disease ${ }^{9}$ (whether this is evident clinically or electrocardiographically) - this association was described more than 70 years ago. For this reason, a recent United Kingdom consensus statement for good practice in the management of hypothyroidism recommends that in older patients, especially those with ischaemic heart disease, the initial dose of thyroxine should be $25 \mu \mathrm{g}$ and increased every three to four weeks by $25 \mu \mathrm{g}$ increments. ${ }^{10}$ There is little evidence that triiodothyronine treatment is better than thyroxine in patients with ischaemic heart disease, although there is a theoretical benefit should angina worsen and thyroid hormone replacement be stopped because the half life of triiodothyronine is shorter than that of thyroxine. While it is reported that up to $15 \%$ of patients starting thyroxine therapy will sustain a myocardial infarction within two years, it is also clear that chest pain improves or resolves in up to half of patients with this problem. ${ }^{11}$ Nonetheless, $40 \%$ of patients with angina are unable to tolerate full replacement doses of thyroxine. ${ }^{12}$ It has been shown that the presence of untreated or partially treated hypothyroidism does not affect adversely the outcome of coronary artery surgery or angioplasty, so there should be a low threshold for proceeding to angiography. In those considered unsuitable for surgery or angioplasty, adequate doses of thyroxine may not be achieved, even if the daily dose of thyroxine is $25 \mu \mathrm{g}$ or less, with gradual incremental increases every few weeks.

\section{Significance of minor abnormalities of thyroid function}

Prevalence studies have shown that "subclinical" thyroid dysfunction (defined biochemically as increases or decreases in serum thyrotrophin concentrations in association with normal circulating thyroxine concentrations) is common in the general population, especially in people older than 60 . Abnormal serum thyrotrophin concentrations are also reported in about half of those in the community prescribed thyroxine long term. We have previously demonstrated that $20 \%$ of patients in the community taking thyroxine have a serum thyrotrophin concentration above the normal range, indicating either poor compliance or inadequate dose prescription; a similar pro- 
portion has suppression of serum thyrotrophin, indicating a degree of over treatment. ${ }^{13}$ The question of whether these biochemical abnormalities are associated with significant cardiovascular consequences, either via direct actions of thyroid hormones on the heart or via changes in the circulating lipid profile, is a controversial issue.

Subclinical hyperthyroidism (low serum thyrotrophin with normal thyroxine) secondary to thyroxine therapy has been reported to be associated with an increase in nocturnal heart rate and electrocardiographic abnormalities such as shortening of the pre-ejection period systolic time interval; a recent study has also demonstrated enhancement of left ventricular systolic function in this condition. ${ }^{14} \mathrm{We}$ and others have also demonstrated in echocardiographic studies that subclinical hyperthyroidism due to thyroxine therapy is associated with an increase in left ventricular mass index, a marker of left ventricular hypertrophy. ${ }^{15}$ In a study recently published in Heart, we reported that thyroxine treatment, in doses that reduced serum thyrotrophin to below normal, had no effect on blood pressure, heart rate, left ventricular systolic function, or stroke volume index, but was associated with an $18.4 \%$ increase in left ventricular mass index compared with controls. ${ }^{15}$

Untreated thyrotoxicosis was associated with a similar effect on left ventricular mass index (in addition to effects on heart rate and systolic blood pressure), but in those patients antithyroid treatment rapidly abolished any difference from controls. ${ }^{15}$ It is notable that the $\beta$ adrenergic blocker bisoprolol has been shown to be effective in abolishing this increase in left ventricular mass index in subjects on thyroxine, as well as abolishing diastolic dysfunction. ${ }^{16}$ The significance of the demonstrated degree of left ventricular hypertrophy in patients prescribed thyroxine long term (and the role of simultaneous $\beta$ blocker therapy) remains unknown, but given the evidence that left ventricular hypertrophy is an independent risk factor for cardiovascular morbidity and mortality, at least in the setting of hypertension, these findings are of concern.

A further area of controversy is the risk of dysrhythmias in patients with subclinical hyperthyroidism. A serial study of 25 patients with hypothyroidism starting thyroxine therapy demonstrated no new onset of significant ventricular or supraventricular arrhythmias. ${ }^{17}$ Nonetheless, a much larger and more recent study based upon the Framingham population in the United States revealed that subjects with a low serum thyrotrophin concentration are at threefold higher risk of developing atrial fibrillation over a 10 year period. ${ }^{18}$ While most of the subjects with low serum thyrotrophin in that population were not taking thyroxine, these findings raise the question of whether subclinical hyperthyroidism secondary to thyroxine therapy is similarly associated with an increased risk of atrial fibrillation, with attendant consequences in terms of cardiovascular morbidity and mortality.

The possible effects of thyroxine therapy on cardiac mass and arrhythmia risk raise concerns regarding adverse influences of mild thyroxine excess on cardiac risk; however, the effect of thyroxine therapy on the lipid profile may confer a beneficial effect on cardiovascular risk. Some studies of thyroxine treatment of mild or subclinical hypothyroidism have shown little or no effect on the lipid profile. ${ }^{6}$ It is notable that our own data have indicated a marked impact of thyroxine treatment on total and low density lipoprotein cholesterol concentrations when sufficient thyroxine is given to suppress serum thyrotrophin to below normal - that is, when subclinical hyperthyroidism is induced. ${ }^{19}$

\section{Thyroid status and cardiovascular morbidity and mortality}

There have been few epidemiological studies of the long term cardiovascular consequences of hypothyroidism and its treatment with thyroxine, especially in doses which reduce serum thyrotrophin to below normal. A study of 29 women treated long term with thyroxine indicated no difference in rates of cardiovascular morbidity or mortality compared with controls. ${ }^{20}$ A larger study of 1180 patients treated with thyroxine (about half of whom had low serum thyrotrophin) revealed an increased risk of hospital admission for ischaemic heart disease among those younger than 65 years, but no difference among those with normal and suppressed thyrotrophin. ${ }^{21}$ The findings from the latter study do not clearly establish that mild thyroid hormone excess was itself the cause of an increase in ischaemic heart disease, not least because half of the subjects included in the study had a previous history of overt hyperthyroidism, which may itself have represented the major risk factor. Nonetheless, these findings, and those summarised above, indicate the need for further epidemiological investigation of the consequences of hypothyroidism and its treatment on cardiovascular risk.

MICHAEL GAMMAGE,

University of Birmingham, JAYNE FRANKLYN

Oueen Elizabeth Hospital,

Edgbaston, Birmingham B15 2TH

1 Parle JV, Franklyn JA, Cross KW, Jones SC, Sheppard MC. Prevalence and follow-up of abnormal TSH concentrations in the elderly in the United Kingdom. Clin Endocrinol (Oxf) 1991;34:77-85.

2 Klein I, Ojama K. Cardiovascular manifestations of endocrine disease. $\mathcal{f}$ Clin Endocrinol Metab 1992;75:339-42.

3 Venkatesh N, Lynch JJ, Uprichard AC, Kitzen JM, Singh BN, Lucchesi BR. Hypothyroidism renders protection against lethal ventricular arrhythmias in a conscious canine model of sudden death. $\mathcal{f}$ Cardiorasc Pharmacol 1991;18:703-10.

4 Bing RF, Briggs RSJ, Burden AC, Russell GI, Swales JD, Thurston H. Reversible hypertension and hypothyroidism. Clin Endocrinol (Oxf) 1980;13:339-42.

5 Forfar JC, Muir AL, Toft AD. Left ventricular function in hypothyroidism. Br Heart F 1982;48:278-84.

6 Roti E, Minelli R, Gardini E, Braverman LE. The use and misuse of thyroid hormone. Endocr Rev 1993;14:401-23.

7 Steinberg AD. Myxedema and coronary artery disease-a comparative autopsy study. Ann Intern Med 1968;68:338-44.

8 Vanhaelst L, Neve P, Chailly P, Bastenie PA. Coronary artery disease in hypothyroidism. Lancet 1967 ;ii: $800-2$.

9 Keating FR, Parkin TW, Selby JB, Dickinson LS. Treatment of heart disease associated with myxedema. Prog Cardiovasc Dis 1961;3:364-81.

10 Vanderpump $M$, Ahlquist J, Franklyn JA, Clayton RN. Consensus statement for good practice and audit measures in the management of hypothyroidism and hyperthyroidism. $B M 71996 ; 313: 539-44$

11 Toft AD. Thyroxine therapy. N Engl F Med 1994;331:174-80.

12 Levine HD. Compromise therapy in the patient with angina pectoris and hypothyroidism: a clinical assessment. Prog Cardiovascular Dis 1961;3: 364-81.

13 Parle JV, Franklyn JA, Sheppard MC. Thyroxine replacement therapy [letter]. Lancet 1991;337:171.

14 Biondi B, Fazio S, Carella C, Amato G, Cittadini A, Lupoli G, et al. Cardiac effects of long term thyrotropin suppressive therapy with levothyroxine. 7 Clin Endocrinol Metab 1993;77:334-8.

15 Ching GW, Franklyn JA, Stallard TJ, Daykin J, Sheppard MC, Gammage MD. Cardiac hypertrophy as a result of long-term thyroxine therapy and thyrotoxicosis. Heart 1996;75:363-8.

16 Fazio S, Biondi B, Carella C, Sabatini D, Cittadini A, Panza N, et al. Diastolic dysfunction in patients on thyroid stimulating hormone suppressive therapy with levothyroxine: beneficial effect of $\beta$-blockade. $f$ Clin Endocrinol Metab 1995;80:2222-6.

17 Polikar R, Feld GK, Dittrich HC, Smith J, Nicod P. Effect of thyroid replacement therapy on the frequency of benign atrial and ventricular replacement therapy on the frequency of benign

18 Sawin CT, Geller A, Wolf PA, Belanger AJ, Baker E, Bacharach P, et al. Low serum thyrotropin concentrations as a risk factor for atrial fibrillaton in older pe

19 Franklyn JA, Daykin J, Betteridge J, Hughes EA, Holder R, Jones SR, et al. Thyroxine replacement therapy and circulating lipid concentrations. Clin Endocrinol (Oxf) 1993;38:453-9.

20 Petersen K, Bengtsson C, Lapidus L, Lindstedt G, Nystrom E. Morbidity, mortality, and quality of life for patients treated with levothyroxine. Arch Intern Med 1990;150:2077-81

21 Leese GP, Jung RT, Guthrie C, Waugh N, Browning MCK. Morbidity in patients on L-thyroxine: a comparison of those with a normal TSH to those with suppressed TSH. Clin Endocrinol (Oxf) 1992;37:500-3. 